\title{
Toward a Blended Ontology: Applying Knowledge Systems to Compare Therapeutic and Toxicological Nanoscale Domains
}

\author{
Christopher M. Grulke, Michael-Rock Goldsmith, and Daniel A. Vallero \\ National Exposure Research Laboratory, US Environmental Protection Agency, Research Triangle Park, NC 27711, USA \\ Correspondence should be addressed to Daniel A. Vallero, vallero.daniel@epa.gov
}

Received 3 October 2011; Accepted 7 January 2012

Academic Editor: P. Bryant Chase

Copyright (C) 2012 Christopher M. Grulke et al. This is an open access article distributed under the Creative Commons Attribution License, which permits unrestricted use, distribution, and reproduction in any medium, provided the original work is properly cited.

\begin{abstract}
Bionanomedicine and environmental research share need common terms and ontologies. This study applied knowledge systems, data mining, and bibliometrics used in nano-scale ADME research from 1991 to 2011. The prominence of nano-ADME in environmental research began to exceed the publication rate in medical research in 2006. That trend appears to continue as a result of the growing products in commerce using nanotechnology, that is, 5-fold growth in number of countries with nanomaterials research centers. Funding for this research virtually did not exist prior to 2002, whereas today both medical and environmental research is funded globally. Key nanoparticle research began with pharmacology and therapeutic drug-delivery and contrasting agents, but the advances have found utility in the environmental research community. As evidence ultrafine aerosols and aquatic colloids research increased 6-fold, indicating a new emphasis on environmental nanotoxicology. User-directed expert elicitation from the engineering and chemical/ADME domains can be combined with appropriate Boolean logic and queries to define the corpus of nanoparticle interest. The study combined pharmacological expertise and informatics to identify the corpus by building logical conclusions and observations. Publication records informatics can lead to an enhanced understanding the connectivity between fields, as well as overcoming the differences in ontology between the fields.
\end{abstract}

\section{Introduction}

The hazard of a chemical compound largely depends on its product formulation (i.e., an impregnated solid suspension versus a chemical solution). Recently, there has been much interest in the role that the size of a particle plays in chemical hazard and exposure potential [1], such as the degree to which very small particles cause greater chemical risk than larger particles with the same chemical composition. Both nanoparticles and derivative nanomaterials are pervasive and growing at a rate of over 243 new products per year worldwide. It is currently estimated that 1317 nanoproducts are manufactured by 587 companies in over 30 countries. These consumer products span several major categories (e.g., health and fitness, home and garden, automotive, food and beverage, crosscutting, electronics and computers, appliances, and goods for children) [2], yet their full impact on product life-cycle and human health still remains poorly understood due to a paucity of harmonized standards for safety, environmental impacts, and human health threats.
In fact, there is still some dispute over what should be deemed a nanoparticle [3]. Current standards in biomedicine and environmental contamination define nanoparticles as being $<100 \mathrm{~nm}$ in at least one dimension. Whereas such dimensional thresholds between nanoscale and bulk materials are useful for research and scientific inquiry, they are not completely applicable to toxicology and risk assessment. When looking at a particle as a whole, its dimensions may not meet the above criteria for a nanoparticle while its construction may have important nanoscale variability that requires it be studied as a nanoparticle. As evidence, take a nanoparticle composite with two distinct compartments: surface and core. These particles may change in time and space, so that the core becomes the surface with time, as the original surface is degraded (e.g., during metabolism and environmental degradation). Such particles constituencies (i.e., surface versus core) are therefore key in determining efficacy and toxicity as a function of time and space, thereby validating the need for studying such a particle using 
nanoscale techniques though its overall dimensions need not be $<100 \mathrm{~nm}$.

Setting the definition of a nanoparticle aside, the federal government of the United States has with the creation of the National Nanotechnology Initiative (NNI-see also: http://www.nano.gov/) identified a need for the organizational framework to coordinate nanorelated research efforts and domains among the federal agencies. The eight major research areas are the following: (1) fundamental nanoscale phenomena and processes, (2) nanomaterials, (3) devices and systems, (4) instrumentation and standards, (5) nanomanufacturing, (6) facilities and acquisitions, (7) environment, health, and safety, and (8) educational and societal dimensions. Interestingly, the most impactful fields of research appear to be aggregated under a single consolidated area: environment, health, and safety. This choice to aggregate such fields is one that should be scrutinized further.

In a sense, researchers advancing therapeutics investigate similar phenomena as researchers aiming to improve the understanding of environmental xenobiotics, but in opposite directions. That is, both assess and address the factors that govern the dose-response characteristic of the material and the risks presented by an agent, whether it is chemical, or physical. However, biomedicine is looking for ways to improve the efficacy of a substance while environmental toxicologists are searching for ways to prevent a xenobiotic from having biological effects.

According to the US Environmental Protection Agency's (EPA) white paper on nanotechnology, the primary research target for absorption, distribution, metabolism, and elimination (ADME) research of nanoparticles is ecological risk (i.e., mainly focused on nonhuman species). In addition, lessons learned from computational toxicology in other agencies such as the U.S. Food and Drug Administration (FDA) or on methods gleaned from natural or incidental particulate matter may prove to be useful to fill the data gaps for these emerging materials [5]. However, such hypotheses cannot be tested without first identifying the landscape of available research. Hence, informatics and other knowledge tools provide a means of assessing the growth, extent, and congruence of these research domains addressing nanomaterials.

Drug delivery concepts can, in theory, be applied to toxicology models, such as the need to keep a substance that is efficacious in certain tissue from reaching other tissues for which it is toxic (i.e., controlled dose, pharmacokinetics, or toxicokinetics). For example, liposomes are developed for drug delivery based on their ability to evade immune response and enabling bioavailability of the drug to the target site. Both the biomedical and environmental scientific communities are seeking better models for pharmacokinetic and dynamic (PBPK/PD) behavior of nanoparticles. These models should be built using the expertise of both contingents as the same ADME processes are in force. Further, emergent materials, including nanoparticles, have attracted considerable attention from scientists from the exposure and health hazards communities alike. The source of these emerging materials in products, and their overall environmental fate and transport, environmental degradation,
TABLE 1: Comparison table of critical factors at play in nanotoxicology versus toxicity of bulk material of identical composition (reproduced from: [4]).

\begin{tabular}{ll}
\hline Bulk properties & Nanoscale properties \\
\hline Chemical composition & Structure (nanostructure) \\
Dose (mass concentration) & Particle concentration \\
Exposure route & Size distributions \\
Reactivity & Particle numbers \\
Conductivity & Aggregation/agglomeration \\
Morphology & Surface adsorb ability \\
Physical form & Surface area \\
Impurities & Surface charge \\
Solubility & Self-assembly \\
& Quantum effects \\
\hline
\end{tabular}

biodegradation, biological uptake and disposition, and effects remains poorly characterized [5].

Usually, the toxicity, ADME, and other hazards associated with chemical ingredients are a function of the physical composition of the substance. This means that the properties of both the surface and the substance core help to determine the chemical risk. For example, surface properties drive the mobility and toxicity of engineered nanomaterials/particle, but the makeup of the material construct that has been coated is also important. To this extent it has become more apparent that there are significantly more than the nine factors commonly used for bulk materials that affect nanomaterial ADME and toxicity. Some of these nanomaterial specific factors are listed in Table 1.

Chemical manufacturers, pharmaceutical companies, and environmental researchers all share interest in the characteristics of nanoparticles that may allow them to "hide" from physiological processes as they find their way to the target cells. For example, nanoparticles for drug delivery consist of various biological substances like albumin, gelatin, and phospholipids for liposomes, and abiotic substances, including various polymers and solid metal containing nanoparticles. The potential interaction of these nanoparticles with tissues and cells is under investigation, but there appears to be both great promise and concern, depending on whether the target is drug delivery or potential toxicity [1]. One aspect of more adequately addressing emerging concerns of nanomaterials is being able to properly characterize both their core and surface properties, variables that ultimately delineate many downstream phenomena found in biological systems that have remained elusive in public health and environmental characterization.

Reliable information is needed to determine whether a nanoparticle may provide benefits, risks, or, as is usually the case, both. In the analogous field of chemical characterization, a typical means of gaining such information is to compare a chemical to other chemicals with similar structures (known as quantitative structure activity relationships (QSAR)). Unfortunately, QSAR treatment of chemicals has its limitations. In order to estimate properties, there must be a sufficient amount of data regarding similar chemicals. More 
importantly, QSAR cannot account for unique differences in even very similar compounds. For example, it is well known that chemicals with identical formulas, but with different configurations (e.g., enantiomers), may either be efficacious or toxic as ingredients in medicines. Likewise, chiral compounds with the same composition but different handedness [6] will have very different environmental persistence (e.g., the right-handed chiral may take years to biodegrade whereas the left-hand compound may degrade in a few weeks). While QSAR methods have shown some applicability in the field of nanoparticle characterization, they certainly are not a complete solution [7].

The typical means of gaining information about ADME, aside from developed QSAR models and other tools for predicting whether a bulk material or compound will present risks, is to conduct in vitro, in vivo, and, more recently, in silico experimental methods. In vitro studies may look at cellular activity in a petri dish whereas in vivo studies may expose rats to the chemical ingredient and observe effects. In silico studies employ computational methods such as genomic and proteomic studies, data mining, molecular modeling, and quantitative structure activity relationships. The pharmacokinetics/pharmacodynamics of a chemical or its modified progeny must be understood in order to determine how the particle and its components become bioavailable. Similarly, a variety of accelerated degradation studies can be performed on varied core-surface combinations by using biomimetics methods (i.e., lung-on-a-chip technology) and employing models tailored for nanomaterial characterization properties such as the chirally perturbed particle in a box model (CPPIB) [8] and other core-surface models. Subsequently, morphology size domain optimization can be completed via microscopy and chromatography specialists and methods. These studies fully integrate a variety of vital activities, research streams, and facilities to take on a major emerging challenge for this novel class of materials, from both a modeling and characterization perspective.

While the high degree of overlap in environmental, health, and safety nanoparticle research promotes the consolidation of the fields into one area, the differences in ontology between the fields is a significant impediment. Often, different terminology is used to describe similar phenomena or homonyms for different phenomena [9]. An example of the former is the terminology employed for very small particles $(<100 \mathrm{~nm}$ diameter). Aerosol researchers call them ultrafines. Nanotechnologists and material scientists call them nanoparticles. Aquatic biologists and chemists call them colloids. Soil scientists call them clay textures. An example of the latter is the " $\mathrm{E}$ " in ADME. Biomedical researchers usually consider it to be excretion whereas environmental toxicologists usually consider it to be elimination. Another example is the use of adsorption by physicists and engineers versus its use by ADME researchers.

Such ontological differences typically lead to greater segregation of researchers and reduce cross-field integration of similar research streams. Such segregation may lead to underutilization or slower incorporation of important discoveries made by specialists in either the health or the environmental specialty of nano research and thereby impede the advancement of nanoscience. In this effort, we investigate the growth of nanoparticle ADME research using bibliometric techniques to visualize nature of growth, gaps in study, and the isolation of research streams in the hope that the pressing issues in the field can be identified leading to a cohesive and comprehensive body of nanoparticle ADME research.

\section{Methods}

2.1. Corpus Selection. To obtain a knowledge concerning the state of research in nanoparticle ADME, we sought to obtain the corpus of relevant peer-reviewed literature by searching two databases: the Web of Knowledge (WoK) and PubMed (http://www.PubMed.org/). These two databases provide a comprehensive collection of peer-reviewed literature citations related to nanoparticle ADME properties. Other databases containing unreviewed publications on the topic were omitted to ensure the integrity of the literature corpus.

Collecting the full set of the literature related to nanoparticle ADME research involved integration of several queries with Boolean logic. Thankfully, such logic is simple to form using the query engines available through the Web of Knowledge (WoK) advanced search interface and PubMed title filters. The primary limitation in accessing the appropriate articles was due to difference in the annotation of articles as being related to ADME. In order to collect a corpus that we felt covered the relevant literature space, we were forced to include several terms related to ADME, pharmacokinetic modeling, or degradation. The inclusion of nano-related terms and toxicity was more facile. The respective queries shown in Table 2 retrieved a total of 1802 and 630 references from WoK and PubMed.

2.2. Bibliometric Techniques. Using the "Analyze Results" section of Web of Science (WoS) found within WoK (Web of Knowledge) various reports (related to WoS subject heading domains, authors, countries, dates, journal titles, etc.) were generated as a csv files for export into various graphing applications. For the sake of this paper, we have exported the reports related to histograms of (a) timeline, (b) WoS subject heading, (c) journal title, and (d) country of origin and have imported (b) and (d) into Many Eyes (http://www.ManyEyes.com/) to create a bubble-plot and a geographical map representation of the data, respectively. Graphs for (a) and (c) were generated in Excel (Microsoft) (see Figures 2-5).

Many Eyes is an open-access Web 2.0 data visualization (or visual analytics toolbox) provided and developed by IBM. We imported the domain-specific corpus of interest into Many Eyes and used the Phrase Net visualization option with Term 1 and Term 2 word structure map as an example (see Figure 6).

Exporting the WOS reference list (plus cited references) as a text file and importing into HistCite, we generated a "histiograph" or timeline that contains information of impact factor (circle size scaled to impact factor) as well as linkages between citations within the specific collection or corpus (see Figure 7). 
TABLE 2: Queries used to obtain the studied literature corpus.

\begin{tabular}{|c|c|c|}
\hline Database & Query & Record count \\
\hline \multirow{5}{*}{$\begin{array}{l}\text { ISI's Web of Knowledge } \\
\text { (http://wokinfo.com/) }\end{array}$} & $\mathrm{TI}=($ nano* $\mathrm{OR}$ nanotox $*)$ AND & \multirow{5}{*}{1802 references } \\
\hline & $\begin{array}{l}\text { TS }=((\text { pharmacokinetic* OR toxicokinetic* OR disposition OR } \\
\text { distribution OR ADME OR pbpk OR environment })) \text { AND TS }=\left(\text { toxic }^{*}\right)\end{array}$ & \\
\hline & Timespan = All Years & \\
\hline & Databases $=$ SCI-EXPANDED & \\
\hline & Lemmatization $=$ On & \\
\hline $\begin{array}{l}\text { PubMed } \\
\text { (http://www.PubMed.org/) }\end{array}$ & $\begin{array}{l}\text { (Tissue distribution OR biodistribution OR dosimetry OR } \\
\text { pharmacokinetics OR dermal OR oral OR inhalation OR uptake OR } \\
\text { absorption OR route OR metabolism OR accumulation OR clearance OR } \\
\text { renal OR hepatic OR excretion OR elimination) AND (nano* OR } \\
\text { ultrafine*) }\end{array}$ & 630 references \\
\hline
\end{tabular}

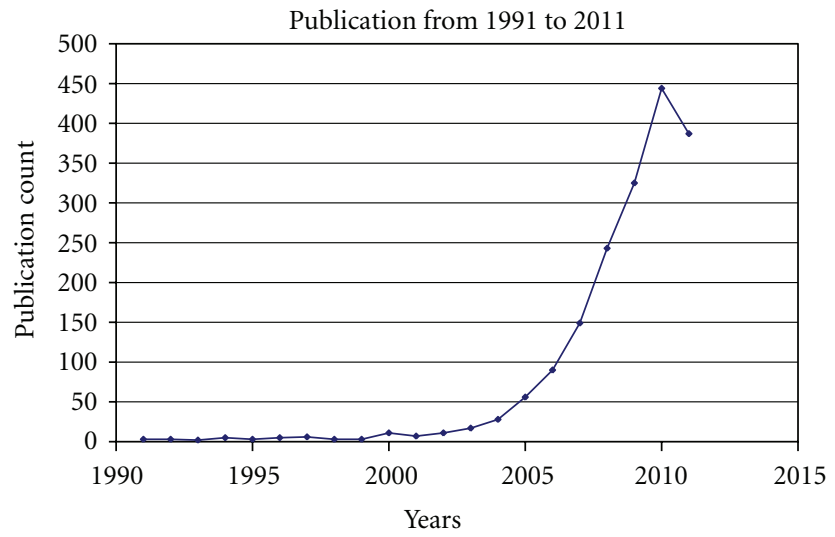

Data source: WOS, ISI Thomson Reuters: 1802 total source: web of knowledge

Query $=\mathrm{TI}=\left(\right.$ nano $^{*}$ OR nanotox $\left.*\right)$ and TS $=(($ pharmacokinetic $*$ OR toxicokineti* OR disposition OR distribution OR ADME OR pbpk OR environment $\left.{ }^{*}\right)$ ) and TS $=($ toxic $*)$ Timespan $=$ All Years.

Data bases $=$ SCI-EXPANDED. Lemmatization $=$ On 1802 records

(1991-2011) 60 records (Before 2002)

FIgURE 1: The distribution by year of the 1802 publications documented in the Web of Knowledge (WoK-see Table 2). The exponential growth continued throughout the decade ending in 2010. The drop in 2011 is an artifact due to partial year reporting.

\section{Results and Discussion}

Bibliometric analysis was carried out on two corpora of the literature resulting from searching of PubMed and the Web of Knowledge (WoK) as described in Section 2.

3.1. Growth of Nanoparticle ADME. Figure 1 delineates the growth in the number of publication related to ADME, toxicity, and nanoparticles within the WoK. It can clearly be seen that a drastic increase in publication started near 2002. In the eleven years prior to 2002, only 60 articles were recorded in the WoK. Since, 1742 articles related to nanoparticle ADME have been documented. To better understand the structure of this growth, we examined the time period before and after this critical point when research started to become mainstream.

We first sought to determine whether the growth evident from Figure 1 was driven in a small number of locations, or if the study of nanoparticle ADME had spread from isolated research centers broadly throughout the world. Figure 2 shows the distribution of publications by country of authorship for publication before and after 2002. While the study of ADME properties of nanoparticles may have originally been primarily segregated to industrialized countries (U.S., Canada, Western Europe, China, and Japan), it has since been picked up throughout the world. A large number of the world's nations have produced research focused on nanoparticle ADME. This global expansion of research highlights the importance of the topic and the general concern regarding nanoparticles.

The global spread of interest in nanoparticles could be caused by either an increased interest in the topics studied prior to the spread or an integration of nanoparticle research into more topics. To determine which one described the observed growth, we examined the subject areas for which ADME nanoscale research was being carried out (Figure 3 ). It is clear that the medical field drove the initial push of nanoparticle ADME research. Pharmaceutical scientists intent on controlling the release of medication pioneered the study of nanoparticle delivery systems. The topic of pharmacology/pharmacy contained over 50\% of all publication prior to 2002. Upon investigating the journals in which this research is commonly published, the importance of the medical field on the inception of nano-ADME became more apparent (Figure 4). While publication is evenly spread amongst the represented journals prior to 2002, all of the journals are related to medical research. However, as this area has matured, environmental sciences and toxicology have shown marked growth. As seen in Figure 3, the number of articles related to these two fields has caught up to the number of publications related to medical applications. In addition, the number of specialty areas and topics has expanded. Both of these aspects of growth are also apparent when looking through the set of journals (Figure 4). Environmental Science \& Technology has become the journal containing the most ADME nanoscale research evoking 


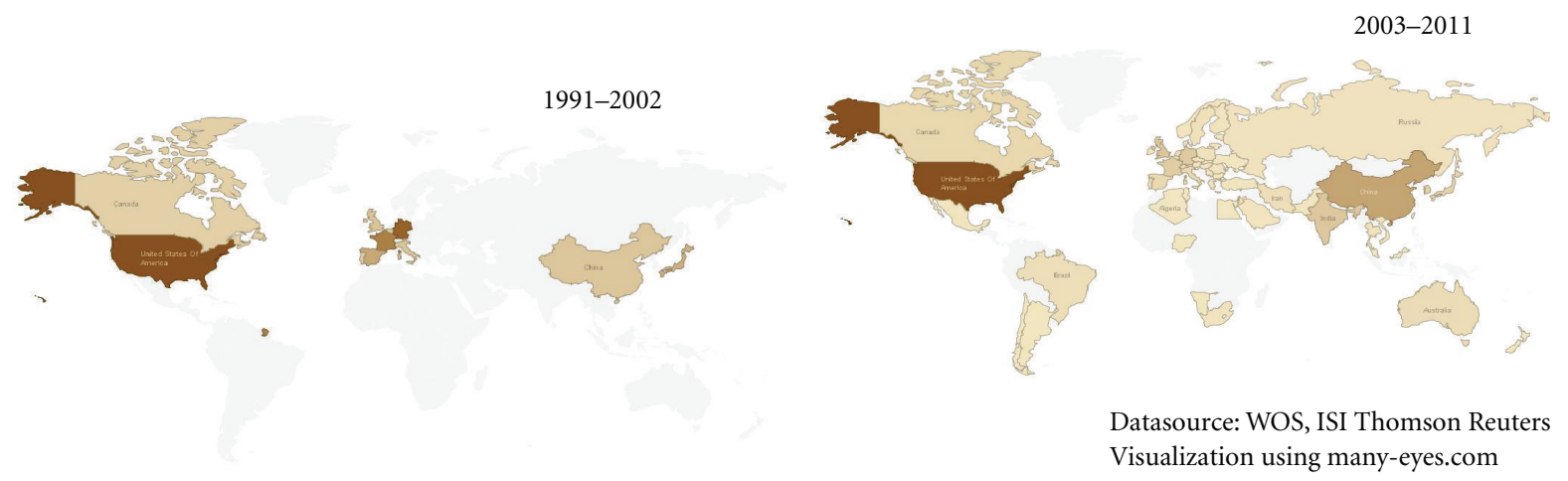

FIgURE 2: The distribution of publications by country of origin in the time before and after the boom of nanoresearch. The maps show record for the years prior to and after 2002. Prior to 2002 the majority of novel nano research was originally centralized in North America and certain pockets of Europe whereas China was a major source for manufacturing. More recently China has become a major player in research (not just manufacturing) and global efforts spanning South America, to portions of Asia and Africa, and Eastern Europe have also become evident alongside North America's effort. (see Table 2: total of 1802 record corpus from WoK analyzed by country of origin in WoK and exported to http://www.ManyEyes.com/).

$1991-2002$

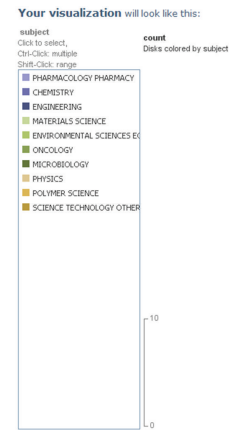

$1991-2002$

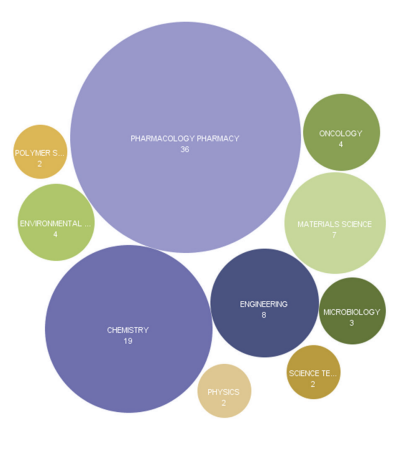

2003-2011

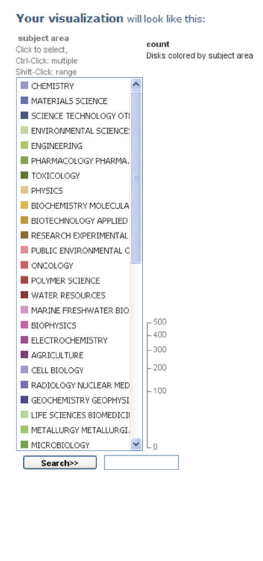

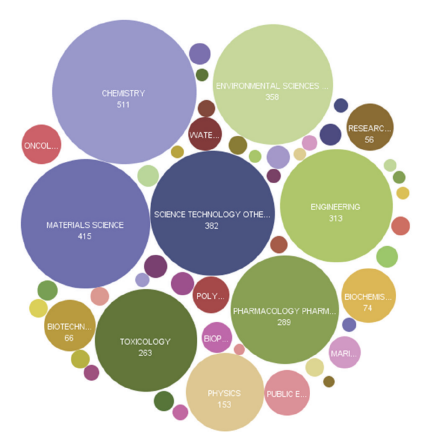

Datasource: WOS, ISI Thomson Reuters Visualization using many-eyes.com

FIGURE 3: The distribution of publications by subject area in the time before and after the boom of nano research (i.e., $~ 2002)$ as classified by WoK subject heading citation analysis of 1802 records (see Table 2).

the former while the increase in specialty journals stresses the latter.

3.2. Key Terms. While it is clear that the study of nanoparticle ADME has grown, we considered it important to understand which physiological processes were being the most examined. By studying the frequency of terms contained within the collected nanoparticle ADME corpus of the literature, insights into the extent of the covered fields were obtained. We sought to uncover such information based on the corpus of the literature we had collected.

First we looked at the frequency of keywords associated with ADME in the literature base. Figure 5 displays these keyword frequencies and highlights important portions of the ADME process that are understudied. Uptake was the most studied part of nanoparticle ADME, particularly uptake via oral and inhalation routes. However, dermal uptake was less studied though this is a highly probable form of environmental exposure. Additionally, the study of nanoparticle uptake has greatly outpaced research into the other parts of the ADME process: metabolism and elimination. This lack of focus on vital elements of the physiological processes responsible for actual dose at targeted tissues points to areas of the field that must be further developed.

Taking the abstracts for our set of articles, we then completed a two-word PhraseNet analysis. The resulting network of terms displayed in Figure 6 provides an excellent summary of concepts within the area of nanoparticle ADME. Key aspects of the research such as engineered properties, exposure types, size domains, key organs, and the underlying research domains are all evident. Such graphics provide a high-level view of the principles connected within the field. It is clear from Figure 6 that the study of the environmental impact of nanoparticles has driven a large part of the increased growth in nanoparticle ADME research, having become the key term centralized in the network. 


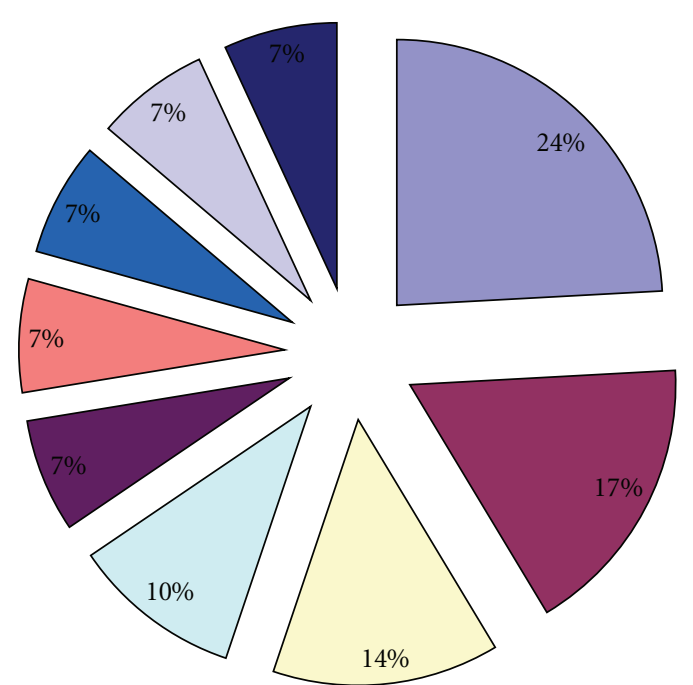

$\square$ International Journal of Pharmaceutics

$\square$ European Journal of Pharmaceutics and

$\square$ Biomaterials

Biopharmaceutics

$\square$ Journal of Controlled Release

๑ Drug Development and Industrial Pharmacy

$\square$ Journal of Microencapsulation

$\square$ Journal of Pharmaceutical Sciences

- Antimicrobial Agents and Chemotherapy

- Pharmaceutical research

2003-2011

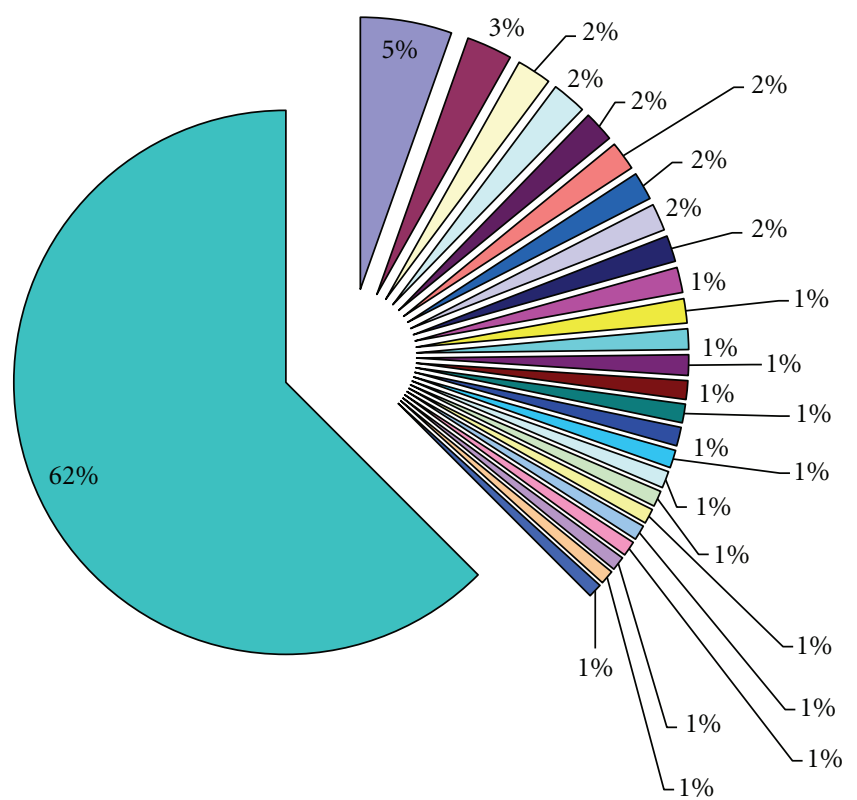

$\square$ Environmental Science Technology

- Journal of Nanoparticle Research

$\square$ Biomaterials

$\square$ International Journal of Pharmaceutics

- Journal of Nanoscience and Nanotechnology

Nanotoxicology

a Nanomedicine

$\square$ Nanotechnology

- ACS nano $\square$ Chemosphere

$\square$ Environmental Toxicology and Chemistry

$\square$ Journal of Biomedical Nanotechnology

- Toxicological Sciences

- Environmental Pollution

Journal of Controlled Release

- Toxicology Letters

$\square$ Journal of Hazardous Materials

Figure 4: The distribution of publications by journal in the time before and after the boom of nano research as classified by WoK publication title analysis of 1802 records (see Table 2). 


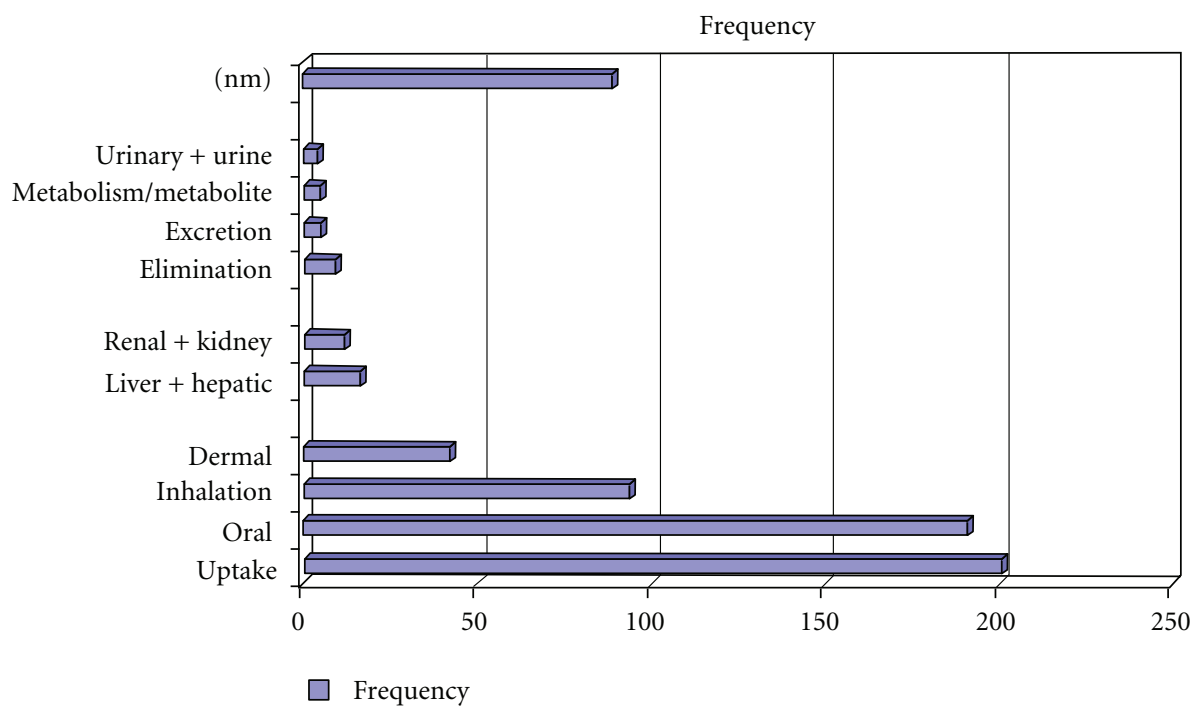

FIGURE 5: Frequency of keywords within the corpus of ADME nanoscale research, as classified by PubMed.org corpus from a custom keyword frequency analysis of 630 records (see-Table 2).

Showing 50 of 2674 terms

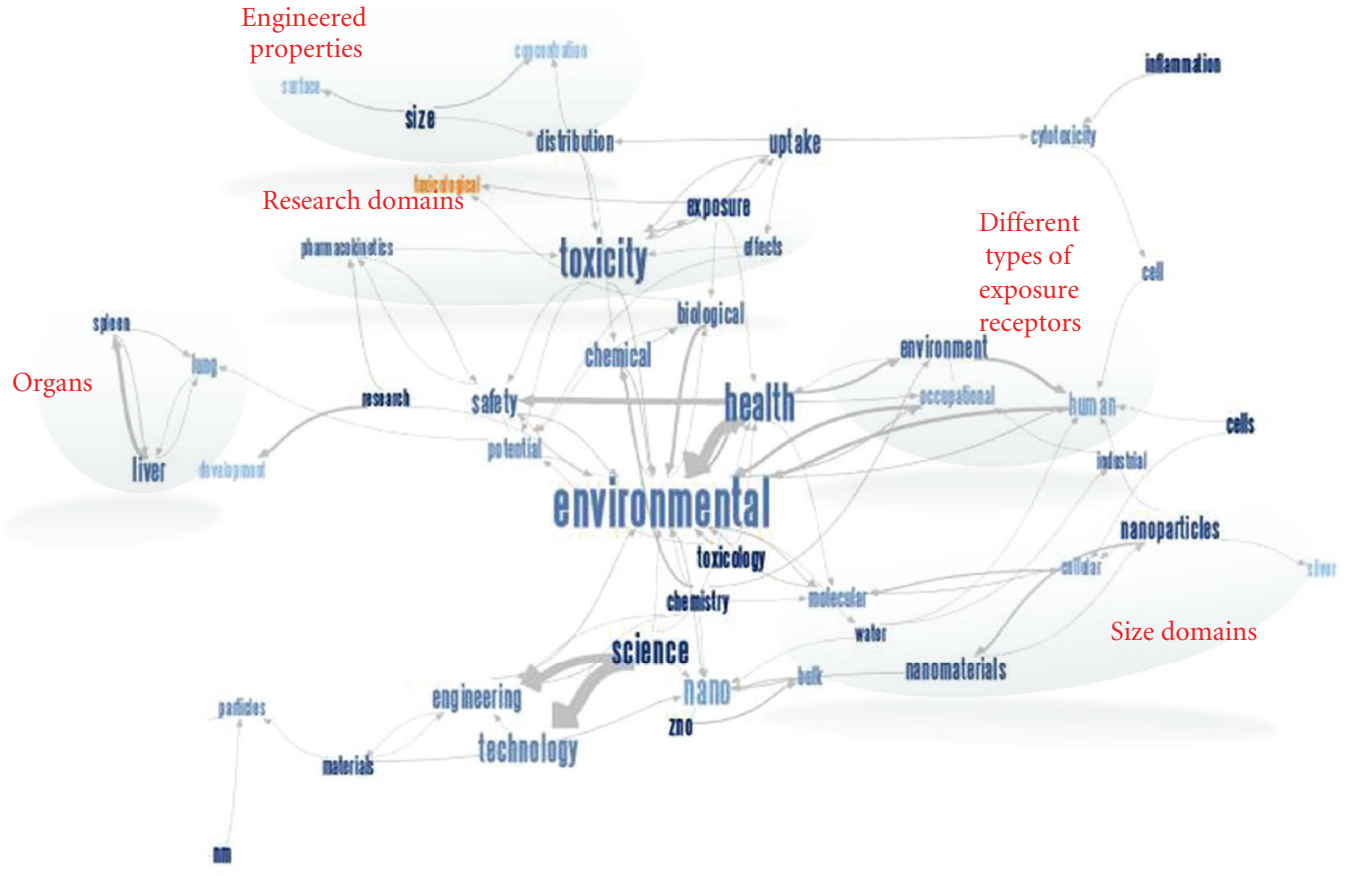

FIgURE 6: Phrase Net generated on the PubMed corpus of nanoparticle ADME publications in http://www.ManyEyes.com/ from a custom keyword frequency analysis of 630 records (see-Table 2). Major themes have been manually circled and annotated.

3.3. Publication Segregation. Unfortunately, the large amount of nanoparticle ADME research does not necessarily mean that it is a cohesive area of research. In fact, the large increase in the number of journals accepting ADME research on nanoparticles (as seen in Figure 4) provides evidence to the contrary. As the field has grown, it has become harder to find and access all the publications that are relevant to the topic. Figure 7 displays the citation analysis of the WoK publication list and indicates segregation has occurred within the corpus of research. While some distinct lines of research are starting to link to other lines, and there may be some pivotal research articles that have both impact factor in addition to memory between years (looking at zoom in of top 23 articles), there is overall a lack of crosstalk between 
Datasource: WOS, ISI Thomson Reuters / visualization histiogram from HistCite:

http://thomsonreuters.com/products_services/science/science_products/a-z/histcite/

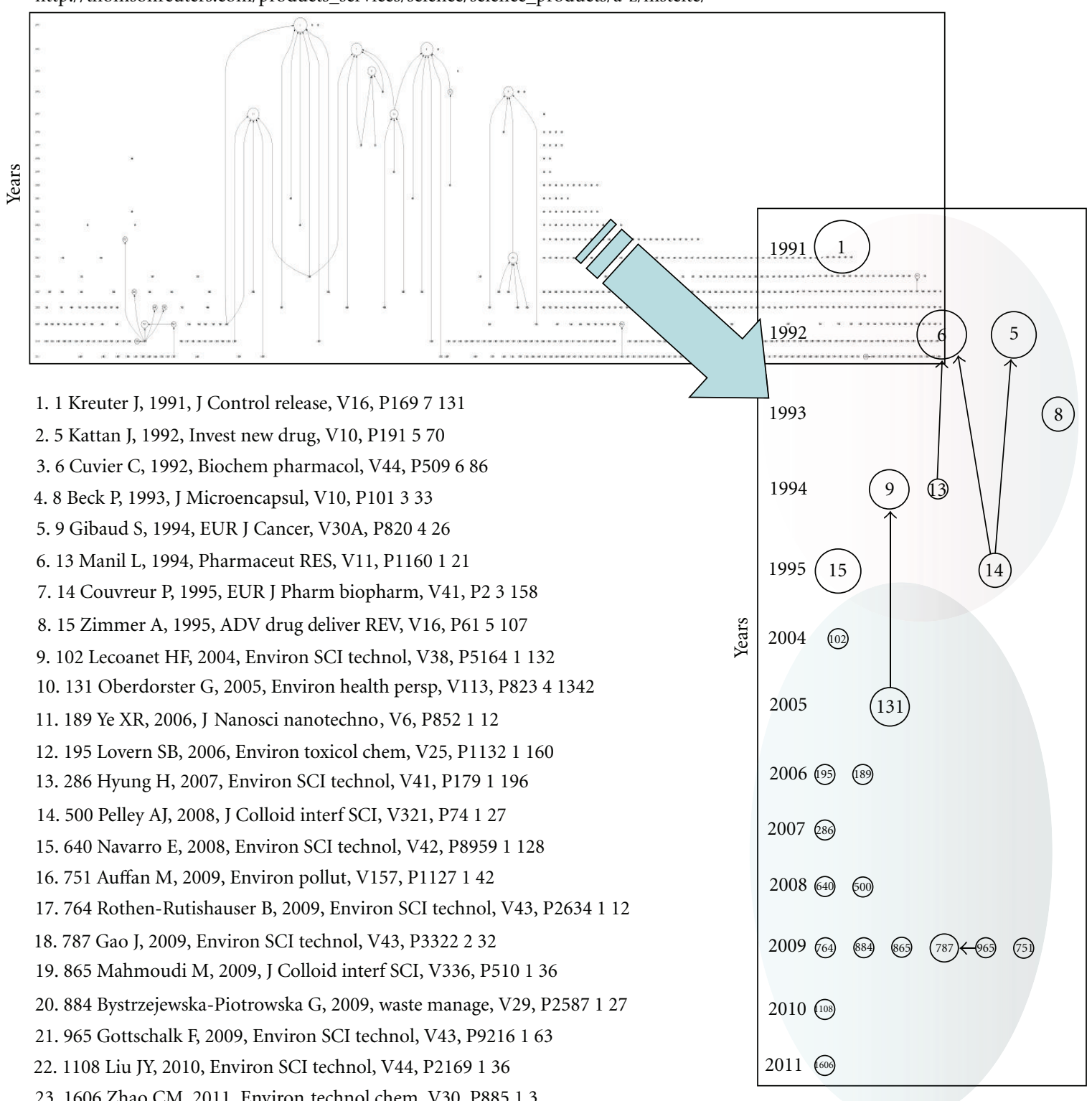

FIGURE 7: Histiograph of WoK corpus of the nano-ADME literature from Table 2, generated using the HistCite package (http://thomsonreuters.com/products_services/science/science_products/a-z/histcite/).

specialties and research efforts. We found it surprising that the majority of high-impact citations connected to none of the previous high-impact citations with the field. What is also apparent from the high-impact internal citation histiograph is that the domains of nanobiomedicine (i.e., therapeutics) and nanotoxicology (adverse effects, and large contribution to environmental characterization) emerged over time. While nanobiomedicine dominated the highimpact citations prior to 2002, the high-impact citations after 2002 were primarily focused on environmental health and nanotoxicology efforts.

\section{Conclusions}

Knowledge tools are effective in identifying trends in research related to nanomaterials [10]. This study described how the research landscape has changed rapidly for health and environmental research. Ten years ago, the medical community dominated ADME nanoscale research almost exclusively. Five years ago, the environmental community surpassed medical research in terms of volume of work and number of journals accepting papers in this area. With the growth of research focused on different aspects for nanoparticle ADME 
and the number of specialty subject in the field, there is a clear need for effective communication amongst researchers. The development of a blended ontology agreed upon by all fields interested in nanoparticle ADME properties could ease this communication.

Based on the key term frequencies, there is a void in the literature that indicates some of the most important aspects of the ADME process for nanoparticles are understudied. We suggest that these areas be addressed more specifically in future research by environmental, health, and safety researchers. In order to reach a point where the pharmacokinetics of nanoparticles are truly understood, study of uptake properties is insufficient.

Finally, this study highlights the segregation of the current literature of what should be compatible academic and professional disciplines engaged in research on this topic. The lack of connectivity within the histiograph of the nanoADME literature seems to indicate that effective communication may not be occurring. It is important to discover whether this is a common aspect of histiographs for other fields of research or a unique feature of nano-ADME research. Further study of the effects of ontology (or lack of consistent language) on research connectivity should be carried out in this field as well as others. While the problem appears to be lessening with key papers connecting the segregated fields, the results lend credence to the hypothesis that current differences in language may be impeding communication, and that blending terminology to avoid ambiguity could enhance collaborations among the health and environmental disciplines.

\section{Disclaimer}

This paper was reviewed by EPA and approved for publication but does not necessarily reflect official agency policy. The appearance or absence of product, services, companies, organizations, home pages, or other websites in this paper does not imply any endorsement by the agency.

\section{References}

[1] W. H. de Jong and P. J. A. Borm, "Drug delivery and nanostructure: applications and hazards," International Journal of Nanomedicine, vol. 3, no. 2, pp. 133-149, 2008.

[2] PEW Charitable Trust, Project on Emerging Nanotechnologies, 2011, http://www.nanotechproject.org/inventories/consumer/.

[3] M. Auffan, J. Rose, J.-Y. Bottero, G. V. Lowry, J.-P. Jolivet, and M. R. Wiesner, "Towards a definition of inorganic nanoparticles from an environmental, health and safety perspective," Nature Nanotechnology, vol. 4, no. 10, pp. 634-641, 2009.

[4] L. Yan, F. Zhao, S. Li, Z. Hu, and Y. Zhao, "Low-toxic and safe nanomaterials by surface-chemical design, carbon nanotubes, fullerenes, metallofullerenes, and graphenes," Nanoscale, vol. 3, no. 2, pp. 362-382, 2011.

[5] U.S. Environmental Protection Agency, Report No. EPA 100/ B-07/001, 2007, http://www.epa.gov/osa/pdfs/nanotech/epananotechnology-whitepaper-0207.pdf.

[6] IUPAC, Compendium of Chemical Terminology, The "Gold Book", Blackwell Science, 2nd edition, 1997, Online corrected version, "chirality", 2006, http://en.wikipedia.org/wiki/Chirality.

[7] D. Fourches, D. Pu, C. Tassa et al., "Quantitative nanostructure-activity relationship modeling," ACS Nano, vol. 4, no. 10, pp. 5703-5712, 2010.

[8] M. R. Goldsmith, C. B. George, G. Zuber et al., "The chiroptical signature of achiral metal clusters induced by dissymmetric adsorbates," Physical Chemistry Chemical Physics, vol. 8, no. 1, pp. 63-67, 2006.

[9] G. M. Whitesides, "The "right" size in nanobiotechnology," Nature Biotechnology, vol. 21, no. 10, pp. 1161-1165, 2003.

[10] D. de la Iglesia, S. Chlesa, J. Kern et al., "Nanoinformatics: new challenges for biomedical informatics at the nano level," Studies in Health Technology and Informatics, vol. 150, pp. 987991, 2009. 

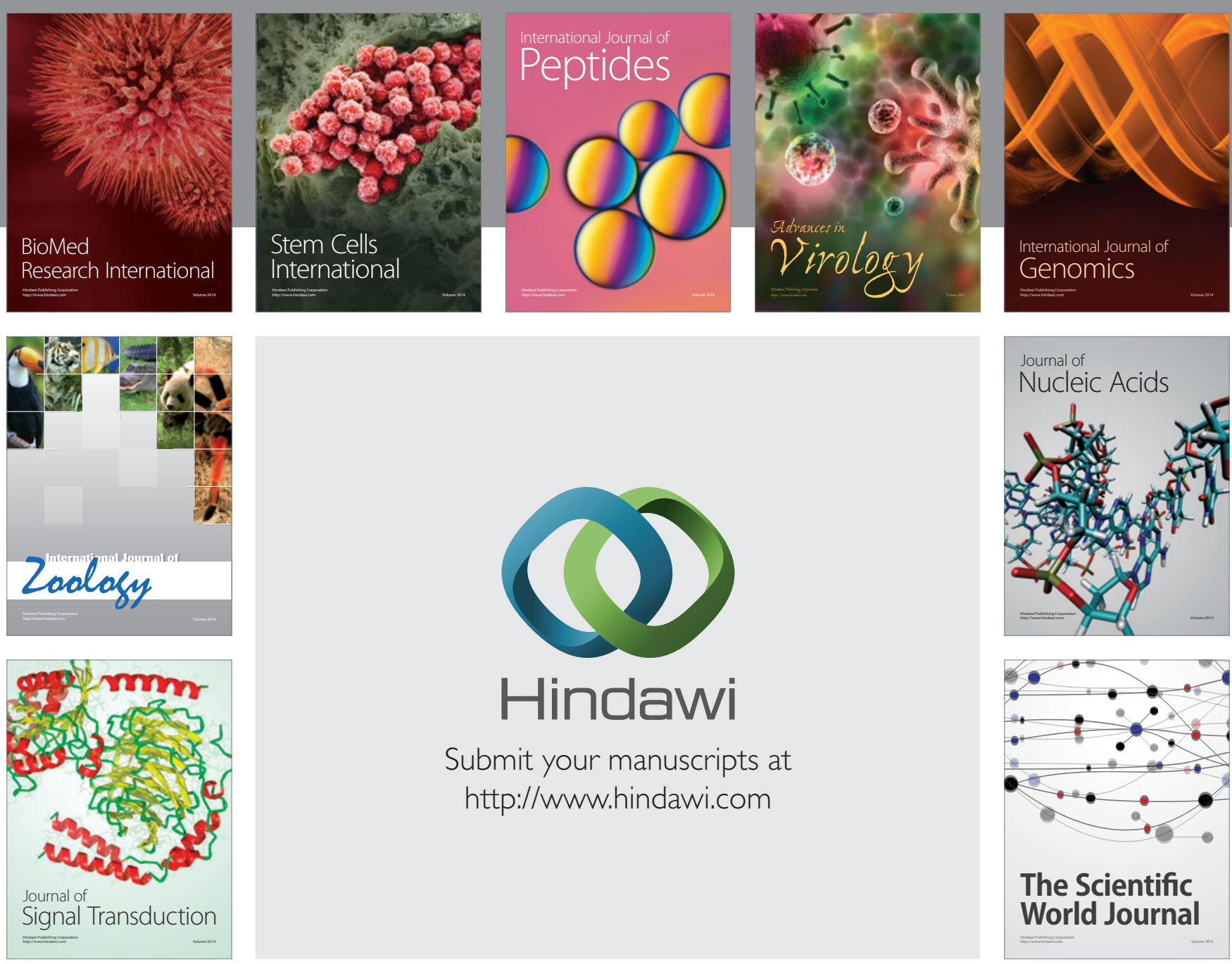

Submit your manuscripts at

http://www.hindawi.com
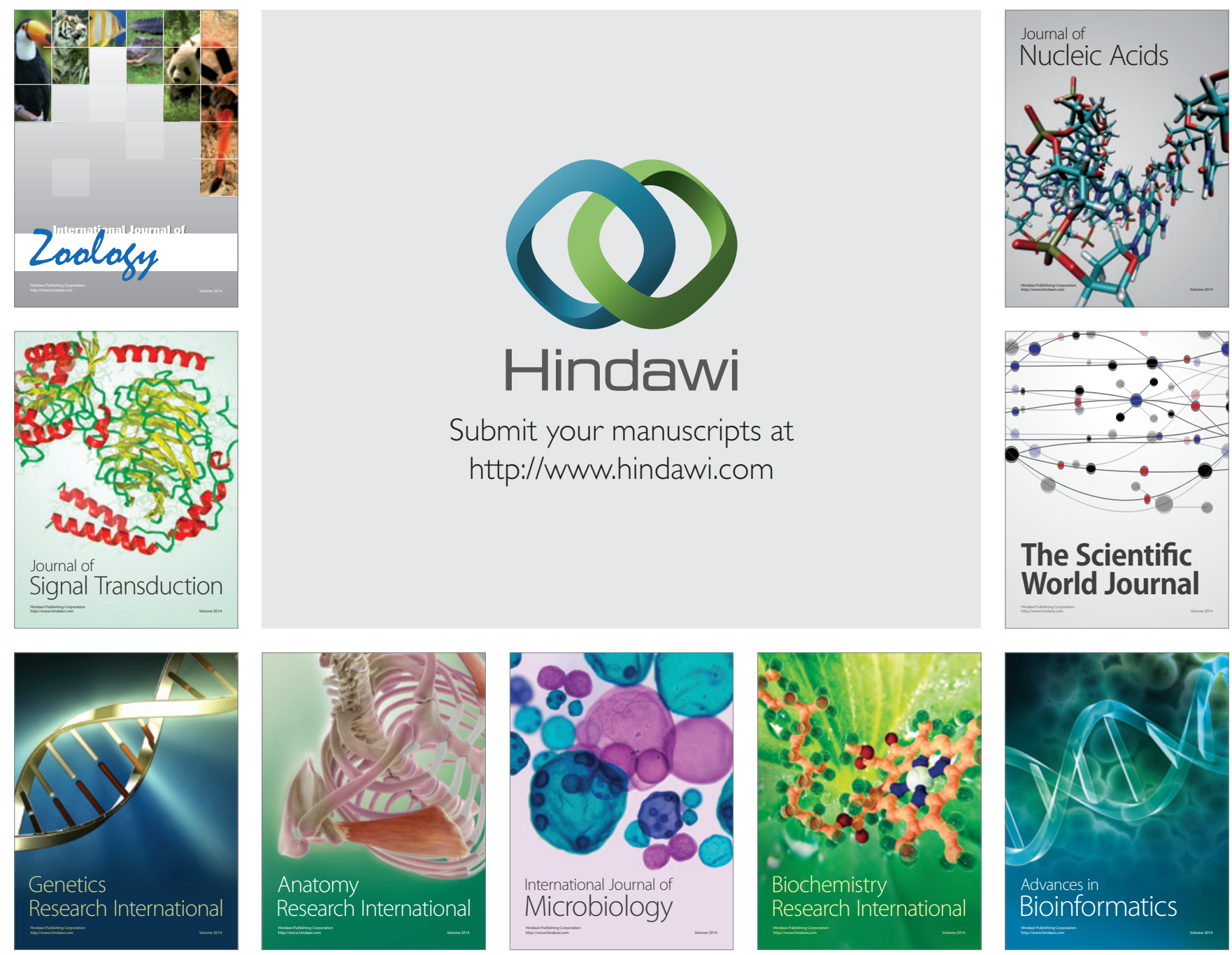

The Scientific World Journal
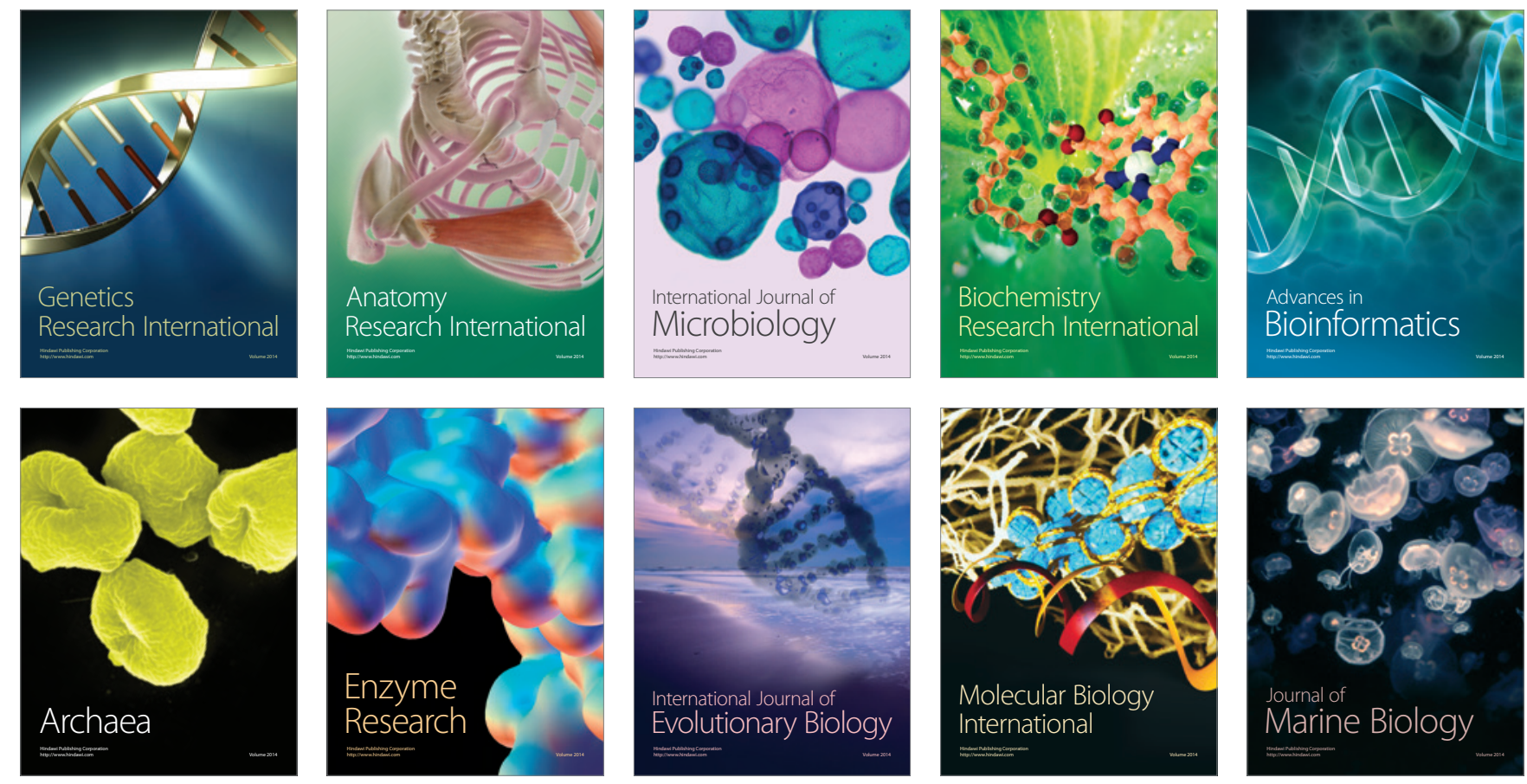